\title{
The Emerging Scholarly Brain
}

\author{
Michael J. Kurtz \\ Harvard-Smithsonian Center for Astrophysics, Cambridge, MA 02138
}

\begin{abstract}
It is now a commonplace observation that human society is becoming a coherent super-organism, and that the information infrastructure forms its emerging brain. Perhaps, as the underlying technologies are likely to become billions of times more powerful than those we have today, we could say that we are now building the lizard brain for the future organism.
\end{abstract}

\begin{abstract}
1. Preamble
"... the highest level of the ant colony is the totality of its membership rather than a particular set of superordinate individuals who direct the activity of members at lower levels."
\end{abstract}

Höldobler and Wilson (1990)

\section{The Super-Organism}

For millennia the only means of intra-species cross-generational communication was chemical; information was transferred via genes in a Darwinian process. The structure for some very complex systems have been transferred by these means.

An example would be the reaction to high temperatures. Even single celled organisms avoid excessively hot regions; those which don't get cooked and don't survive. Fast forward a few eons and we have cooperative structures of 50 trillion cells (e.g. humans) which can very rapidly self mobilize to move a finger away from a frying pan.

A second example, indicating just how complex this chemical communication channel can be is that primates (including humans) are innately afraid of snakes (Hebb, 1955). To quote Pinker (1997) "People dread snakes without ever having seen one. After a frightening or painful event, people are more prudent around the cause, but they do not fear it; there are no phobias for electrical outlets, hammers, cars, or air-raid shelters." Ancient creatures which had an insufficient fear of snakes became lunch rather than becoming our ancestors. The agglomeration of this genetically transmitted information has been called the "collective unconscious" (Jung 1935).

Creatures can learn, bees for example, can learn which color flowers are the best sources of food, and they can communicate, as bees do with their "waggle dance," communicating the distance 
and relative position (to the sun) of a food source. The information needed to perform this dance appears to be entirely genetically transmitted, there is no evidence for a learned component (von Frisch 1967).

Humans, certainly, are capable of learning communication techniques. The development of language, aided by Darwinian processes (Pinker 1994) represents a phase transition in the information transfer process across time which is evolution. It became possible for parents to tell their children "eat this mushroom, but not that one!"

This phase transition enabled humans to form large coherent groups where information could be maintained and shared, thus problems could be solved by distributed efforts. Over time sophisticated bodies of knowledge were built.

The next phase transition in the information transfer process was the development of writing. While oral transmission is powerful, and has provided us with, as an example, the core ideas behind religion, modern civilization is clearly too complex to be achieved without writing.

Writing, along with the two major enabling inventions, paper and printing, has formed the basis for the information transfer system for our globally connected human society. This society, viewed as an entity onto itself, a super-organism, has capabilities far beyond those of single individuals. An example of these capabilities might be the industrial revolution, which was designed and implemented without any central direction.

We are living in another period of phase transition, one that will happen much faster than the previous ones. The time span for phase transitions has been getting shorter by about a factor of 100 each time; implying that the current one will be consummated within a single human lifetime.

The technical underpinnings for the current phase transition are very rapid communication (beginning, perhaps, with the telegraph) and electronic storage. Together our electronic communication and memory network now form a coherent entity, a Super-Brain (Heylighten and Bollen, 1996) for the super-organism which is human society.

Although pure communications networks have existed substantially longer (telegraph, telephone, radio, television) it is the addition of electronic memory which makes the computer network the fundamental technical advance. Within the past few decades computer networks have coalesced to form a single entity (in graph/network studies forming the giant component is usually viewed as a phase transition, Newman 2003).

With this the human society super-organism has developed the functional equivalent of a brain, but we are clearly just at the beginning of the process. In the past 60 years the power of the information technologies behind the transition has increased by a factor of (about) a trillion; if this can continue for another 60 years, or indeed (Kurzweil 2001) increase more rapidly the evolving super-organism might well be incomprehensible to us today.

The exponential growth of technical capabilities is not guaranteed, Moore's law, for example, 
appears to have reached its physical limits (in the number of transistors on a chip). The nanobio-cyber convergence currently occurring suggests, however, that the growth will continue, or, following Kurzweil (2001) accelerate.

The nature of the changes are still in the realm of science fiction; Stapelton (1930) foresaw the fate of the "last men", after which (evolutionarily) humans became a new race:

"The system of radiation which embraces the whole planet, and includes the million million brains of the race, becomes the physical basis of a racial self....

But chiefly the racial mind transcends the minds of groups and individuals in philosophical insight into the true nature of space and time, mind and its objects, cosmical striving and cosmical perfection....

For all the daily business of life, then, each of us is mentally a distinct individual, though his ordinary means of communication with others is "telepathic." But frequently he wakes up to be a group-mind....

Of this obviously, I can tell you nothing, save that it differs from the lowlier state more radically than the infant mind differs from the mind of the individual adult, and that it consists of insight into many unsuspected and previously inconceivable features of the familiar world of men and things."

\section{Architecture}

It appears that we humans, like the Krell (MGM 1956), will be (or indeed are) increasingly interconnected with an electro-mechanical system of almost unimaginable power. Already we communicate near telepathically with almost anyone on the planet, at almost any time, via cell phone; already our memories are tremendously augmented by internet search engines.

While we cannot know exactly what the future will bring, we can intuit some things concerning the elements of our new environment, based on past trends. The elements we construct will be extraordinarily complex; each with a multitude of design decisions. This is unlike the exact sciences, where there is a single correct description of a physical reality; it is more like architecture, there is no single exactly correct bridge or building design.

Certainly there are architectural elements which persist, such as a key stone in building arches. Perhaps the closest analog to the information entity we are now constructing, (the super-brain?) is a city. Cities are constructed by the long term and large scale combined efforts of people. Cities are not explicitly designed, but they grow as a result of numerous mostly independent efforts. Even without explicit design cities normally have a number of design elements in common, such as neighborhoods, or sidewalk cafes (Alexander, et al 1977).

Major elements in all cities are places to get things. These are often the end points of complex 
webs of activity. An example could be a bakery, which combines efforts by fertilizer providers, seed salesmen, farmers, millers, truckers, and others to provide bread. Major elements in the information structure we are building are places to get information. These also are the end points of complex webs of activity. An example could be the Smithsonian/NASA Astrophysics Data System (ADS; Kurtz, et al 1993; 2000; 2005). The ADS combines the efforts of lens designers, rocket scientists, astronomers, publishers and others to provide astrophysics research information.

There are substantial differences in how these design elements are instantiated. There can be bakeries, hardware stores, dress shops, toy stores, etc; and there can be Wal-Mart or Target. Both systems have their advantages. In the world of information there can be specialized providers, such as ADS, PubMed, The Internet Movie Database, Flightstats, etc; and there can be Google or Bing. Again both systems have advantages.

\section{Vectors of the Mind}

Context is an important criterion; asking to get one's tank filled will get an altogether different result at Diver Jim's Belmont Scuba Co than it will if asked at the gas station next door. Likewise the query "plasma diagnostics" will get a different result on PubMed than on ADS.

Even when one can narrow down a request, for a product, or information, to a specific realm, context is still important. A typical hardware store, for example, will have hundreds, if not thousands of different fasteners (nails, screws, glue, tape, ...); which product to purchase depends critically on exactly what the current problem is. Likewise the ADS has thousands of papers on a topic such as "weak lensing", which paper to read depends critically on the exact current problem.

Context is usually provided by people. A customer chooses to go to a hardware store (instead of, say, a cheese shop) to solve some problem, which is described to a clerk. The clerk has substantial expertise in the specific problem domain, and suggests one, or several possible solutions, taking into account the actual needs and abilities of the customer.

In the information world the customer still chooses between providers, ADS or PubMed, for example, but there is no equivalent to the clerk; this function is performed by the information service. Because the actual needs and abilities of each "customer" vary greatly for the exact same query (a beginning student will often be better served by different information than would be best for a world renowned expert) the information system will need to be able to adapt to, and perhaps sense, the larger context of queries. 


\subsection{Global Measures}

\subsubsection{Linear Algebra Techniques}

Clearly information systems perform functions which were previously performed by humans, or by groups of humans. This requires techniques to model the thoughts and behavior of people and groups of people. Psychologists and Sociologists have been developing these techniques for decades.

Thurstone (1934) used eigenvector techniques to build an orthogonal vector space model for human thought. These techniques have become very widely used; psychometrics and marketing for example depend heavily on them. They have also been used to model the idea space of documents. Ossorio (1966) used a psychological testing approach to model individual opinions concerning the relevance of documents. Kurtz (1993) reverse engineered a set of classified documents to build a model of the thought process of a librarian classifier. Perhaps the most successful of these methods is latent semantic analysis (LSA; Deerwester, et al 1990) which builds the vector space based on the co-occurrence of words in documents.

These eigenvector techniques have also been used in many different classification problems; such as with astronomical spectra. Kurtz (1982) first applied the method to stellar spectra, and Connolly, et al (1995) rediscovered it for galaxy spectra. While the methods have been used, they have not found great success, compared to partitioning line ratio diagrams (Baldwin, et al 1981) or color-magnitude diagrams (Golay, et al 1977). This is likely because, while the techniques are linear, the underlying physics is highly non-linear (Kurtz 1982).

Human thought is also highly non-linear; representing it by a linear vector space is bound to cause problems. Although the success of LSA as an indexing method for text demonstrated that very local measures of nearness can be effectively defined, more generally these are not metric spaces. As an example, human perceptions do not follow the Schwartz or triangle inequality. The perceptual distance between a dog and a vacuum cleaner is made shorter by the introduction of a third point, a mechanical dog; a mechanical dog is a kind of dog, and a mechanical dog is a kind of vacuum cleaner (Kurtz 1989).

\subsubsection{Social Network Techniques}

By analyzing the connections between entities (people, documents, molecules, traffic jams, ...) one can build powerful descriptive and predictive models (Barabasi 2003, Newman 2003). Many of the techniques were developed for the social sciences (Wassermann and Faust 1994) and are now

widespread. Fifteen percent of the papers published in 2009 by Physical Review E address aspects of network problems, as do twenty-six percent of papers published in PLOS Computational Biology, for example. 
The structure of information networks contains information about the intelligent processes which created them. Obtaining the underlying structure from the network is called community detection, and is similar to techniques for cluster analysis or classification.

Fortunato (2010) reviews these methods, currently the most popular method is that of Girvan and Newman (2002) and the "best" (according to Lancichinetti and Fortunato 2009) is that of Rosvall and Bergstrom (RB: 2008). RB have used their algorithm on citation data to show the interrelationships between the major fields of science; their map may very profitably be compared with the similar map of Bolen et al (2009a) who show a similar structure based on usage data and pre-existing field classifications.

The RB algorithm has been used by Kurtz, et al (2007) and Henneken et al (2009) to map the subfields of astronomy, based on both citation data and on shared keywords for journal articles. Attempts to build a similar map from usage data have not, thus far, been successful, perhaps because of the very broad readership patterns of many astronomers.

Another measure obtainable from a network graph is the "importance" of the individual nodes. Importance is normally called centrality in this context, and there are several different centrality measures. In a friendship network, where people are the nodes and they are linked to other people by friendship degree the person with the most friends would be the person with the highest degree centrality. Note that friendship is directional, I may consider you my friend, but that does not mean that you consider me a friend; thus the concepts of in-degree and out-degree. In a citation network the paper with the highest in-degree is the most cited paper, while review articles would have very high out-degree.

Betweenness centrality is another "importance" measure. In a friendship network the most central people are those with friends in many different, otherwise autonomous cliques; in a journal to journal citation network the most central journals are the interdisciplinary journals (Leydesdorff 2007), like Science or Nature, which are between otherwise autonomous fields, such as astronomy and neuroscience. Betweenness centrality is a key measure in an information flow network; the high betweeness centrality nodes facilitate information transfer between fields.

The currently most used centrality measure is the expected occupation time for each node when visited by a random walk, where the agent randomly follows links from node to node. This is normally called eigenvector centrality, as the result is the same as the first eigenvector of the node-node connectivity matrix (Bonacich 1971). Google's famous Page-Rank algorithm (Brin and Page 1998) is essentially eigenvector centrality, with clever implementation details.

There are several other centrality measures. Kurtz and Bollen (2010) give a brief introduction; Koschützki, et al (2005) a detailed discussion. 


\subsection{Local Measures}

While measures which solve for globally optimum measures are clearly desirable and useful, they are often not feasible. This is especially true in situations where the data is inherently highly multipartite. Journal articles, for example, may be connected by citations, but they may also be connected by having the same author, or having been read by the same reader, or concerning the same subject matter, or the same astronomical object, or ....

An interesting question is how much is lost if one uses local measures, instead of attempting a global solution. Some recent work discusses this.

Eigenfactor (West, et al 2010a) is a useful measure of scholarly journals; it is available in the Thompson-Reuters Web of Science, and finds frequent use by librarians in making purchasing decisions. The Eigenfactor is essentially eigenvector centrality, measured on the journal to journal citation graph.

Davis (2008) pointed out that the Eigenfactor journal rankings in a subfield (medicine is what Davis used) are very similar to rankings derived from simple citation counts. In their two dimensional comparison of 39 different network measures Bollen et al (2009b) showed eigenvector centrality near to in-degree, but not identical. West et al (2010b) showed convincingly that the Eigenfactor produces rankings which are significantly different from plain citation counts.

While eigenvector centrality is clearly different from simple in-degree, and in cases such as Eigenfactor likely better, the fact remains that the differences are small. This suggests that in many instances very similar results may be had using much simpler measures.

The ADS second order operators (SOO: Kurtz 1992; Kurtz et al 2002) make use of local measures in the multipartite space to achieve specific results. The SOO are not a ranking or clustering methodology, per se, rather the SOO are relational operators which one uses to build custom rankings. Two examples of their use, both taken from the ADS Topic Search (adsabs.harvard,edu/cgibin/TopicSearch), will serve as an illustration. Here we will use the language of networks, Kurtz, et al (2005) use the language of lists of attributes. Both examples find information about a technical topic, while this can be almost anything, for concreteness we'll use the topic "weak lensing."

Example 1 starts by taking the article to article citation graph, where articles (nodes) are (directionally) connected by citations (links). First we take the subset of nodes which concern the topic, weak lensing; next we sort the nodes in the subset by their in-degree centrality, and retain only the top N (say 200). Next we form a super-node which is the sum of the individual top 200 nodes, and we sort the entire graph on the out-degree centrality to the super-node. The top of this list are articles which cite a large number of articles on weak lensing which are, themselves, highly cited. In other words we have found review articles on weak lensing.

Example 2 starts with the article-reader bipartite graph, where an article is connected to a reader if that person has read that article. Again we initially restrict the articles to ones concerning 
the topic weak lensing, then we sort that list on an attribute of the article, but now that attribute is the publication date, we take the most recent 200. Again we form a super-node with the 200 articles, and sort the reader nodes by out-degree to this super node. We take the readers with high out-degree to the article super-node (persons who have read recent papers on weak lensing) and form a reader super-node. Finally we sort all the articles in the (non restricted) graph on in-degree from the reader super-node. The top of this list are the currently most popular papers among persons working in the field of weak lensing.

Notice how these constructions solve many of the problems associated with trust (Josang, et al 2007). Only directly relevant, and well regarded papers and individuals are used; ADS further restricts the readers by removing persons who come from external search engines, who usually do not share the goals of professional researchers (Kurtz and Bollen 2010).

The SOO were first implemented in the ADS in 1996; they are used throughout the system, and form much of the basis for the myADS notification service (myads.harvard.edu).

\subsection{Recommender Systems}

If direct queries to an information system, such as ADS, can be viewed as consciously recalling data from the collective memory, then recommender systems might be likened to having memories pop into your head. Recommender systems as a research and application field cover an enormous range of different areas (c.f. recsys.acm.org) focusing mainly on commercial uses; here we will concentrate on the recommender systems for scholarly literature.

In a scholarly field, such as astrophysics, the information is denser, and the use sparser, than in many other fields. For example The Videohound's Golden Movie Retriever lists about 30,000 movies; the ADS contains more than four times this number of papers which contain the word cosmology in the abstract. The peak usage for a scholarly article is usually the first day it becomes available (so there can be no usage information) and decreases rapidly. The typical ten year old article from a major journal is downloaded once per month.

The ADS is building a set of recommenders based on the article currently being read, in the future we will also create recommenders based on the article viewing history of the reader. The exact details are yet to be determined, the systems use nearly all the techniques described in this section, as well as the CLUTO (Zhao and Karypis 2004) clustering software. Kurtz, et al (2009) and Henneken, et al (2010) describe the initial implementation; which is intended for use by active researchers.

Briefly we use the text and references in an article to find a set of recent articles which are very similar to the target article in subject matter. These articles are then used with the SOO to find recommendations using the words/keywords, citations, usage, authors, and astronomical objects. 


\section{The Scholarly Brain}

In addition to having immensely enhanced memories, we now also have immensely enhanced perception. The new data intensive science (Gray 2007) rests not simply in the mechanical extension of our perception, as begun by Galileo and van Leeuwenhoek, but on the automated perception and analysis of huge data sets. The ATLAS experiment at CERN has a raw data rate of $60 \mathrm{~TB} / \mathrm{s}$ (Klous 2010), about fifty trillion times the information handling capacity of humans (Fitts 1954).

The underlying technologies are being developed to satisfy the needs of huge systems, like the LHC experiments, but ever larger systems of sensor networks are being created to take advantage of the new capabilities. For these systems to combine their impact, they need to be able to communicate with each other; they need to share a language (Kurtz 1989; 1992). The shared language need not be native to any particular system or experiment (Hanisch 2001), but needs to be universally understood, like medieval Latin, or English today. This standardization is taking place across all scientific and technical fields, examples are the International Virtual Observatory Alliance's Simple Application Messaging Protocol (SAMP; Taylor, et al 2009) or the Open Access Initiative's Object Re-use and Exchange (OAI-ORE) protocol (Van de Sompel, et al 2009).

Automated memory and automated perception are combining to form automated ideas. Standardized data descriptors along with semantic tagging of text (Accomazzi 2009) produce a new environment, in which inference engines, similar to the stochastic/syntactic procedures used to analyze bubble chamber tracts (Fu and Bhargava 1973) are able to discover new, important associations and patterns.

These systems will be able to model and predict the behavior of humans (Barabasi 2010), indeed Ossorio $(1967,1977)$ spent a good fraction of his career using his methods to model the behavior of specific humans. The role of these systems will not be to model individual human behaviors, but will be to act as the functional core for our collective intelligence.

This collection of machines will not provide the totality of the brain for the emergent superorganism which is human society; the higher functions, the cognitive layer, will be provided by the sum of all people. The machines will provide the core functionality, the lizard brain. Analogous with our own evolution there will be innate capabilities, like our instant reaction to heat, and anomalies, like our fear of snakes.

Individual scholarly disciplines have long functioned as semi-autonomous super-organisms. The memory function (on paper) being journal articles in university libraries. With the advent of the internet scholars are rapidly transforming their work habits to take advantage of the possibilities (Borgman 2007). New technologies effecting the speed of information transfer (Ginsparg 1994), and the ability to directly and effectively access huge datasets (Szalay and Gray 2001) have already been created, more are coming daily. The scholarly literature has been fully digital for more than

a decade. The idea that a discipline, such as astronomy, already functions as a coherent superorganism, with electronic memory and perception systems is not at all far fetched. 
Millions of years before he imagined it would occur we are beginning to implement Stapledon's(1930) racial mind, beginning first not with the human race, but with the sub races of astronomers, bio-chemists, economists, particle physicists, etc. With this scholarly brain we are achieving "insight into many unsuspected and previously inconceivable features of the familiar world of men and things."

\section{Acknowledgments}

This essay is dedicated to the memory of two extraordinary scientists who showed me great

kindness. Peter Ossorio's work continues to inspire me in several ways. Jim Gray's (2007) $4^{\text {th }}$ Paradigm is the clearest exposition of how the new science will actually function.

Also Andre Heck has, through the years, provided me, and many others, with venues to discuss the deeper meaning of some current trends. The references to this paper alone list several.

The ADS is funded by NASA Grant NNX09AB39G.

\section{REFERENCES}

Accomazzi, A. (2010) Astronomy 3.0 Style. ArXiv e-prints arXiv:1006.0670.

Alexander, C., Ishikawa, S., and Silverstein, M. (1977) A Pattern Language, New York: Oxford University Press.

Baldwin, J. A., Phillips, M. M., Terlevich, R. (1981) Classification parameters for the emission-line spectra of extragalactic objects. Publications of the Astronomical Society of the Pacific 93, $5-19$.

Barabasi, A-L (2003) Linked, New York: Plume.

Barabasi, A.-L. (2010) Bursts: the hidden pattern behind everything we do, New York: Dutton.

Bollen, J., Van de Sompel, H., Hagberg, A., Bettencourt, L., Chute, R., Rodriguez, M.A.,Balakireva, L. (2009a) Clickstream Data Yields High-Resolution Maps of Science, PLoS ONE, 4, e4803

Bollen, J., Van de Sompel, H., Hagberg, A., Chute, R., Mailund, T. (2009b). A Principal Component Analysis of 39 Scientific Impact Measures. PLoS ONE 4, e6022.

Bonacich, P. (1972) Factoring and weighting approaches to status scores and clique identification, Journal of Mathematical Sociology 2, 113-120.

Borgman, C.L. (2007) Scholarship in the Digital Age: Information, Infrastructure, and the Internet, Cambridge: MIT Press. 
Brin, S. \& Page, L. (1998), The Anatomy of a Large-Scale Hypertextual Web Search Engine, Computer Networks and ISDN Systems, 30, 107.

Connolly, A. J., Szalay, A. S., Bershady, M. A., Kinney, A. L., Calzetti, D. (1995) Spectral Classification of Galaxies: an Orthogonal Approach. The Astronomical Journal 110, 1071.

Davis, P.M. (2008) Eigenfactor: Does the Principle of Repeated Improvement Result in Better Estimates than Raw Citation Counts?, Journal of the American Society for Information Science and Technology, 59, 2186.

Deerwester, S., Dumais, S., Furnas, G., Landauer, T., \& Harshman, R. (1990). Indexing by latent semantic analysis. Journal of the American Society for Information Science, 41, 391.

Fitts, P.M. (1954) The Information Capacity of the Human Motor System in Controlling the Amplitude of Movement, Journal of Experimental Psychology 47, 381-391.

Fortunato, S. (2010) Community detection in graphs. Physics Reports 486, 75-174.

Fu, K.S. and Bhargava, B.K. (1973) Tree Systems for Syntactic Pattern Recognition, IEEE Transactions on Computers 22, 1087-1099.

Ginsparg, P. (1994) First steps towards electronic research communication, Computers in Physics 8, 390-396.

Girvan, M., Newman, M. E. J. (2002) Community structure in social and biological networks. Proceedings of the National Academy of Science 99, 7821-7826.

Golay, M., Mandwewala, N., Bartholdi, P. (1977) Spectral classification of stars with the same colours in intermediate multiband photometry - The concept of photometric 'star-box'. Astronomy and Astrophysics 60, 181-194.

Gray, J. (2007) Jim Gray on eScience: A Transformed Scientific Method, in The Fourth Paradigm, Data-Intensive Scientific Discovery, eds T. Hey, S. Tansley, and K. Tolle, Redmond, Washington: Microsoft Research (2009).

Hanisch, R. J. (2001) ISAIA: Interoperable Systems for Archival Information Access. Virtual Observatories of the Future 225, 130.

Heylighten, F. and Bollen, J. (1996) The World-Wide Web as a Super-Brain: from metaphor to model. in Cybernetics and Systems '96 (ed. R. Trappl), p. 917, Vienna: Austrian Society for Cybernetics.

Hebb, D. O. (1955) Drives and the C.N.S. (Conceptual Nervous System), Psychological Review 62, 243-254. 
Henneken, E. A., Accomazzi, A., Kurtz, M. J., Grant, C. S., Thompson, D., Bohlen, E., Murray, S. S., Rosvall, M., Bergstrom, C. (2009) Exploring the Astronomy Literature Landscape. Astronomical Society of the Pacific Conference Series 411, 384.

Henneken, E. A., Kurtz, M. J., Accomazzi, A., Grant, C., Thompson, D., Bohlen, E., Di Milia, G., Luker, J., Murray, S. S. (2010) Finding Your Literature Match - A Recommender System. ArXiv e-prints arXiv:1005.2308.

Höldobler, B. and Wilson, E.O. (1970), The Ants, Cambridge: Belknap Press.

Josang, A, Ismail, R. and Boyd, C (2007) A survey of trust and reputation systems for online service provision, Decision Support Systems 43, 618

Jung, C.G. (1935) Über die Archetypen des kollektiven Unbewussten, Zürich: Rhein-Verlag.

Klous, S, for the ATLAS collaboration (2010) Event Streaming in the Online System: Real-Time Organization of Atlas Data, report number ATL-DAQ-PROC-2010-017, Geneva: CERN.

Koschützki, D., et al (2005) Centrality Indices, in Network Analysis Methodological Foundations, eds U. Brandes and T. Erlebach, Lecture Notes in Computer Science 3418, Heidelberg: Springer.

Kurtz, M. J. (1982) Automatic spectral classification. Ph.D. Thesis, Hanover: Dartmouth College.

Kurtz, M. J. (1989) Classification and knowledge. in Knowledge Based Systems in Astronomy, Lecture Notes in Physics 329, 91-106.

Kurtz, M. J. (1992) Second Order Knowledge: Information Retrieval in the Terabyte Era. in Astronomy from Large Databases II, European Southern Observatory Conference and Workshop Proceedings 43, 85.

Kurtz, M. J. (1993) Advice from the Oracle: Really Intelligent Information Retrieval. in Intelligent Information Retrieval: The Case of Astronomy and Related Space Sciences, Astrophysics and Space Science Library (ASSL) 182, 21.

Kurtz, M. J., Karakashian, T., Grant, C. S., Eichhorn, G., Murray, S. S., Watson, J. M., Ossorio, P. G., Stoner, J. L. (1993) Intelligent Text Retrieval in the NASA Astrophysics Data System. in Astronomical Data Analysis Software and Systems II 52, 132.

Kurtz, M. J., Eichhorn, G., Accomazzi, A., Grant, C. S., Murray, S. S., Watson, J. M. (2000) The NASA Astrophysics Data System: Overview. Astronomy and Astrophysics Supplement Series 143, 41-59.

Kurtz, M. J., Eichhorn, G., Accomazzi, A., Grant, C. S., Murray, S. S. (2002) Second order bibliometric operators in the Astrophysics Data System. Society of Photo-Optical Instrumentation Engineers (SPIE) Conference Series 4847, 238-245. 
Kurtz, M. J., Eichhorn, G., Accomazzi, A., Grant, C. S., Demleitner, M., Murray, S. S. (2005) Worldwide Use and Impact of the NASA Astrophysics Data System Digital Library. Journal of the American Society for Information Science and Technology 56, 36.

Kurtz, M. J., Henneken, E. A., Accomazzi, A., Bergstrom, C., Rosvall, M., Grant, C. S., Thompson, D., Bohlen, E., Murray, S. S. (2007) Mapping The Astronomy Literature. Bulletin of the American Astronomical Society 38, 808

Kurtz, M. J., Accomazzi, A., Henneken, E., Di Milia, G., Grant, C. S. (2009) Using Multipartite Graphs for Recommendation and Discovery. ArXiv e-prints arXiv:0912.5235; to appear in Astronomical Data Analysis Software and Systems XIX.

Kurtz, M. J., Bollen, J. (2010) Usage Bibliometrics. Annual Review of Information Science and Technology, 44, 3-64.

Kurzweil, R. (2001) The Law of Accelerating Returns, www.kurzweilai.net/articles/art0134.html

Lancichinetti, A., Fortunato, S. (2009) Community detection algorithms: A comparative analysis. Physical Review E 80, 056117.

Leydesdorff, L. (2007) Betweenness centrality as an indicator of the interdisciplinarity of scientific journals. Journal of the American Society for Information Science and Technology, 58, 1303-1319.

Metro-Goldwyn-Mayer (1956) Forbidden Planet (film)

Murtagh, F. and Heck, A. (1987) Multivariate Data Analysis, Berlin: Springer Verlag.

Newman, M.E.J. (2003) The structure and function of complex networks, SIAM Review 45, 167.

Ossorio, P.G. (1965) Classification Space - A Multivariate Procedure for Automatic Document Indexing and Retrieval, Multivariate Behavioral Research 1, 479.

Ossorio, P. G. (1967). Attribute space development and evaluation (RADC-TR-67-640), Rome, NY: Rome Air Development Center.

Ossorio, P.G. (1977) "What Actually Happens", The Representation of Real-World Phenomena, Colombia, South Carolina: University of South Carolina Press.

Pinker, S. (1994) The Language Instinct, New York: William Morrow.

Pinker, S. (1997) How the Mind Works, New York: W. W. Norton.

Rosvall, M., Bergstrom, C. T. (2008) Maps of random walks on complex networks reveal community structure. Proceedings of the National Academy of Science 105, 1118-1123.

Stapelton, O. (1930) The First and Last Men, London:Methuen. 
Szalay, A. and Gray, J. (2001) The World-Wide Telescope, Science 293, 2037-2040.

Taylor, M., Boch, T., Fitzpatrick, M., Allan, A., Paioro, L., Taylor, J., Tody, D. (2009) SAMP Simple Application Messaging Protocol, v 1.11, International Virtual Observatory Alliance

Thurstone, L.L. (1934) Vectors of the Mind, Psychological Review 41, 1.

Van de Sompel, H., Lagoze, C., Nelson, M. L., Warner, S., Sanderson, R., Johnston, P. (2009) Adding eScience Assets to the Data Web. ArXiv e-prints arXiv:0906.2135.

von Frisch, K. (1967) The Dance Language and Orientation of Bees, Cambridge: Harvard University Press.

Wassermann, S., and Faust, K. (1994) Social Network Analysis, Cambridge (U.K.): Cambridge University Press.

West, J.D., Bergstrom, T.C., Bergstrom, C.T. (2010a) The Eigenfactor Metrics (TM): A Network Approach to Assessing Scholarly Journals, College and Research Libraries, 71, 236.

West, J., Bergstrom, T., Bergstrom, C.T. (2010b) Big Macs and Eigenfactor Scores: Don't Let Correlation Coefficients Fool You, Journal of the American Society for Information Science and Technology, to appear: (DOI: 10.1002/asi.21374)

Zhao, Y. and Karypis, G. (2004) Empirical and Theoretical Comparisons of Selected Criterion Functions for Document Clustering, Machine Learning, 55, 311-331 